\title{
Novel Oral Targeted Mini Gene-Vaccine Platform Resists Influenza A Challenge
}

\section{Mansour Mohamadzadeh*}

Department of Infectious Diseases and Pathology, Division of Hepatology/Gastroenterology and Nutrition, Emerging Pathogens Institute (EPI), University of Florida, Gainesville, FL 32601, USA

Seasonal influenza remains a formidable threat and kills $250,000-$ 500,000 individuals each year. This threat has been amplified by the recent emergence of a new influenza $\mathrm{A}(\mathrm{H} 1 \mathrm{~N} 1)$ virus that is mediating a worldwide pandemic. Influenza virus infections impact the health of more than $5-15 \%$ of the population worldwide each year, resulting in significant human suffering and economic burden. Influenza infection affects individuals of all ages; however the elderly and children are particularly vulnerable and increased mortality and morbidity is usually observed in these populations [1]. The genome of influenza A viruses is comprised of eight single-stranded, negative sense RNA segments encoding one or two proteins. The surface proteins of the virus, hemagglutinin (HA) and neuraminidase (NA), can be classified into 16HA subtypes (H1-H16) and 9 NA subtypes (N1-N9). HA is the key molecule that binds to its receptor expressed on the cells resulting in viral fusion with the endosomal membranes. Replication and transcription of viral RNAs is accomplished by a heterotrimer of the polymerase subunits, PB2, PB1, and PA, and the nucleoprotein (NP). Subsequently, synthesized viral ribonucleoprotein (vRNP) complexes are transported from the nucleus to the cytoplasm via the nuclear export protein (NEP) and the M1 matrix protein whereupon they are assembled into virions at the plasma membrane. The NA initiates viral release from infected cells by cleaving sialic acids from cellular and viral HA and NA subunits.

Influenza vaccination can provide effective resistance to viral challenge. Although influenza vaccines and antiviral drugs are currently available, they have limited efficacy. Furthermore, the global community is not well prepared for the emergence of pandemic strains of influenza. The production of a vaccine to a newly emerging strain can take 3-6 months-providing a window of time for the virus to spread and substantially burden health care systems throughout the world. Most influenza vaccine strategies target high-risk individuals, such as the elderly. However, these vaccines have been shown to elicit only weak immune responses in the elderly. In addition, viral antigenic drift resulting in a mismatch between the vaccine and the circulating virus may be a concern, as this can lead to inefficacious vaccine outcome. Studies show that the effectiveness of influenza vaccination is limited by the functional quality and breadth of the immune response and the time required for vaccine delivery [2].Thus, there is an urgent need for improved vaccines. To explore new avenues of an efficacious targeted vaccine strategy called mini gene-platform against microbial challenge (i.e., influenza A),we developed 12-mer peptides derived from a phage display peptide library that specifically bind to professional antigen presenting dendritic cells(DCs), with the goal of using these peptides to target antigens specifically to DCs. Such a DC-peptide targeting strategy with peptide expression by gut commensal bacteria, including Lactobacillus acidophilus or Lactobacillus gasseri, increases the bioavailability and immunological potency of delivered immunogenic T cell epitopes.

We have previously used this strategy to elicit robust protective immunity against $B$. anthracis and breast cancer challenge [3-5]. To further extend the potential of such a targeted mini-vaccine, we used this DC-peptide strategy to deliver the highly immunogenic sequences of influenza A (i.e., H1N1 and H3N2) comprised of HA, NA, PA, NS2, PB1, PB2, HP, and NP subunits to intestinal DCs in order to elicit robust $\mathrm{T}$ cell immune responses against influenza infection. The overall emphasis was to develop a DC-based mini gene-vaccine strategy expressed and delivered mucosally by L. gasseri, a beneficial bacterium of the normal gut microbiota that, at the same time, serves as a potent adjuvant which induces the activation of DCs to result in robust Th1 polarization [6]. It is expected that this advantageous mucosal influenza targeted mini gene vaccine should be well tolerated by recipients and is therefore easily delivered to a large susceptible population. The notion of such a mini gene-vaccine platform was to combine the advantages of the orally administered probiotic bacterium with specific targeting of the influenza immunogenic $\mathrm{CD}^{+}$and $\mathrm{CD} 8^{+} \mathrm{T}$ cell epitopes to $\mathrm{DCs}$ in order to elicit potent immune responses against viral challenge. Accordingly, we investigated the oral delivery of this unique targeted mini gene platform expressed by L. gasseri, which is generally regarded as safe for oral consumption. Furthermore, high concentrations of viable L. gasseri expressing the targeted mini genes of $\mathrm{HA} / \mathrm{NA} / \mathrm{PA} / \mathrm{HP} / \mathrm{NP} / \mathrm{NS} / \mathrm{PB}$ fused to our unique DC-peptide can be efficiently produced in a fermenter, concentrated, lyophilized to a powder, and stored for extended periods of time without significant losses in viability. The overall goal of our research efforts was to establish a platform for vaccination against influenza A strains whereupon two novel approaches were combined a) improving vaccine potency by specifically targeting the selected $\mathrm{T}$ cell epitopes of various influenza subunits to intestinal DCs; and b) expressing such a selected mini gene platform in a probiotic strain that serves not only as a potent adjuvant, but also as an effective oral delivery vehicle. With this in mind, we then hypothesized that targeting highly immunogenic epitopes from the HA, NA, PA, HP, NP, NS, and $\mathrm{PB}$ proteins of $\mathrm{H} 1 \mathrm{~N} 1 / \mathrm{H} 3 \mathrm{~N} 2$ will efficiently induce both humoral and cellular immune responses against the viral challenge. This hypothesis was then tested via oral inoculation of L. gasseri expressing the mini gene DC-peptide fusion protein.

To establish such an oral targeted vaccine we first optimized the codon sequences of the mini gene-flu vaccine platform that includes a variety of $\mathrm{CD}^{+}$and $\mathrm{CD} 8^{+} \mathrm{T}$ cell epitopes, such as HA/NA/PA/HP/ $\mathrm{NP} / \mathrm{NS} / \mathrm{PB}$, of $\mathrm{H} 1 \mathrm{~N} 1$ and $\mathrm{H} 3 \mathrm{~N} 2$ influenza A strains [7]. The mini geneDC peptide fusion was then cloned in a heterogonous vector suitable

*Corresponding author: Mansour Mohamadzadeh, Department of Infectious Diseases and Pathology, Division of Hepatology/Gastroenterology and Nutrition, University of Florida, Gainesville, FL 32601, USA, Tel: (352) 294-4117; E-mail: m.zadeh@ufl.edu

Received October 20, 2012; Accepted October 22, 2012; Published October 24 2012

Citation: Mohamadzadeh M (2012) Novel Oral Targeted Mini GeneVaccine Platform Resists Influenza A Challenge. J Vaccines Vaccin 3:e112 doi:10.4172/2157-7560.1000e112

Copyright: (c) 2012 Mohamadzadeh M. This is an open-access article distributed under the terms of the Creative Commons Attribution License, which permits unrestricted use, distribution, and reproduction in any medium, provided the original author and source are credited. 
Citation: Mohamadzadeh M (2012) Novel Oral Targeted Mini Gene-Vaccine Platform Resists Influenza A Challenge. J Vaccines Vaccin 3:e112. doi:10.4172/2157-7560.1000e112

for expression in L. gasseri [5]. Subsequently, groups of mice $(\mathrm{n}=5)$ were vaccinated for four consecutive weeks, rested for two weeks, and boosted for another two weeks with $L$. gasseri harboring an empty vector, L. gasseri expressing mini gene-Flu vaccine fused to a control peptide (FluVac-Ctrl), or L. gasseri expressing mini gene-Flu vaccine fused to DC-binding peptide (FluVac-DC). One week after the last vaccine booster, mice were sacrificed and lymphocytes were isolated from the lungs, draining mesenteric lymph nodes (MLNs), and spleens. Single cell suspensions were prepared and lymphocytes were incubated with currently available PA and NP tetramers in order to identify NP and PA specific CD8 ${ }^{+} \mathrm{T}$ cells. Cells were then stained for their surface expression of CD3, CD8, and CD44 and analyzed by flow cytometry (BD Canto II). Our results indicated that our FluVac-DC vaccine elicits an increase in the percentage of live NP and PA specific $\mathrm{CD}^{+}{ }^{+} \mathrm{CD} 8^{+} \mathrm{T}$ cells in the MLNs and spleens of mice when compared to FluVac-Ctrl and empty vector treated mice (data not shown). Lymphocytes from the spleen and MLNs were then restimulated with NP and PA peptides in vitro in order to determine the production of pro-inflammatory cytokines (i.e., IFN $\gamma, \mathrm{TNF} \alpha$ ) and Granzyme B by live NP and PA specific $\mathrm{CD}^{+}{ }^{+} \mathrm{CD}^{+} \mathrm{T}$ cells. Data demonstrated that $\mathrm{CD} 44^{\text {hi }} \mathrm{PA}$ and NP specific $\mathrm{CD}^{+}{ }^{+} \mathrm{CD} 8^{+} \mathrm{T}$ cells from FluVac-DC vaccinated mice produced increased

A
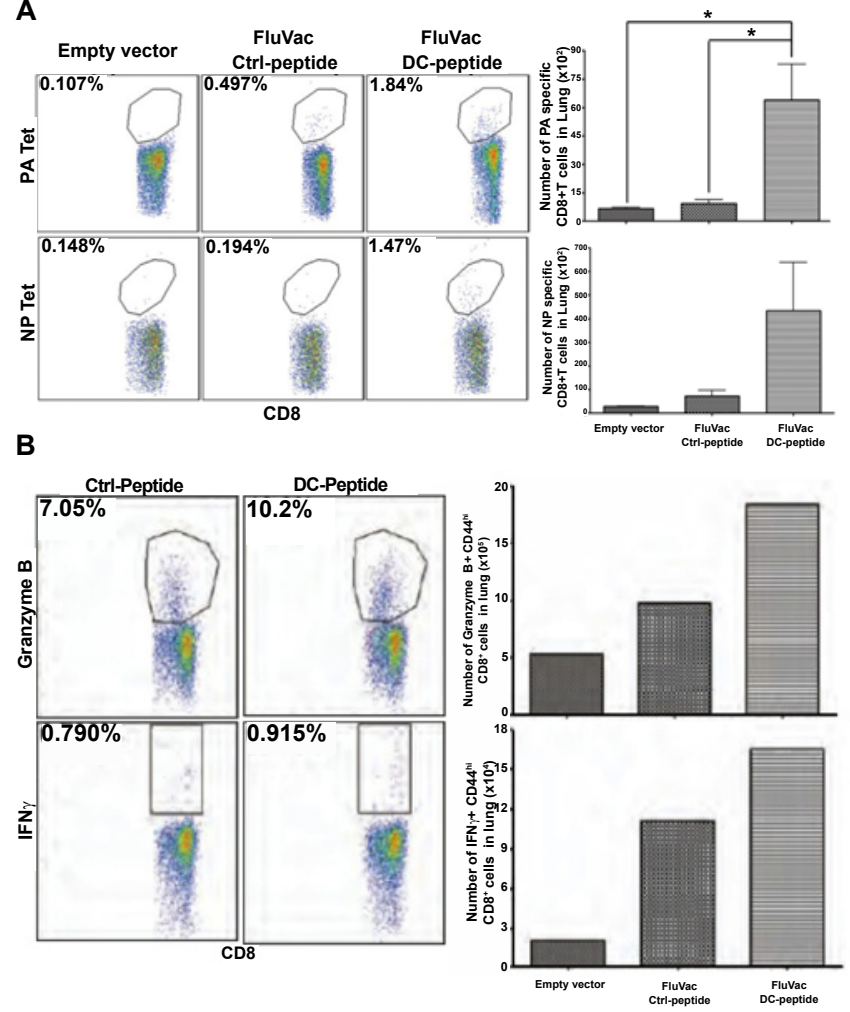

Figure 1: Induction of antigen specific $C D 8^{+} T$ cells in the lungs by $L$. gasseri expressing targeted mini genes of influenza A subunits. Groups of C57BL/6 mice ( $n=5 /$ group) were vaccinated with $L$. gasseri harboring empty vector, FluVac-Ctrl, or FluVac-DC $\left(10^{9} \mathrm{cfu} / \mathrm{mouse}\right)$ for four consecutive weeks. One week after the last boost, mice were sacrificed and lymphocytes were isolated from the lungs that were perfused with PBS/Heparin solution (20U of Heparin/1 mL PBS) by collagenase digestion. 1A) Lung lymphocytes were incubated with live/dead stains to identify live cells, Fc block, and then PA and NP tetramers in order to identify NP and PA specific $C D 8^{+} \mathrm{T}$ cells. $1 \mathrm{~B}$ ) Cells were subsequently stained for their surface expression of CD3, CD8, and CD44, fixed, permeabilized, and intracellularly stained for IFNy, TNFa (data not shown), and Granzyme B and analyzed by FACS Canto. Experiments were performed at least three times with similar trends.
IFN $\gamma$, TNFa and Granzyme B (data not shown). We also analyzed the impact of the mini gene vaccine platform on all $\mathrm{CD} 3^{+} \mathrm{CD} 4^{+} \mathrm{CD} 44^{\text {hi }} \mathrm{T}$ cells. Similarly, $\mathrm{CD} 4^{+} \mathrm{T}$ cells in FluVac-DC vaccinated mice produced significantly more IFN $\gamma$, TNF $\alpha$ and Granzyme B than FluVac-Ctrl or empty vector vaccinated mice (data not shown).

Subsequent experiments were conducted in order to delineate the impact of such targeted mini gene-vaccination on $\mathrm{CD} 4^{+}$and $\mathrm{CD} 8^{+} \mathrm{T}$ cells in the lungs, the primary site of influenza infection. Lungs were perfused with PBS/Heparin solution (20U of Heparin/1 mL of sterile PBS) and lymphocytes were recovered by collagenase digestion. FluVac-DC vaccinated mice generated increased total numbers of NP and PA specific $\mathrm{CD}^{+} \mathrm{T}$ cells in the lungs (Figure 1A). Additionally, restimulated lymphocytes from the lungs of FluVac-DC vaccinated mice produced significantly more IFN $\gamma$, TNFa (data not shown), and Granzyme B than FluVac-Ctrl or empty vector vaccinated mice (Figure 1B). In parallel, groups of mice vaccinated with the same regimens of empty vector, FluVac-Ctrl, or FluVac-DC were infected with A/HK$\mathrm{x} 31$ (x31, H3N2=A/Hong) influenza A virus [8,9] two weeks after the last booster. These studies clearly show the efficacy of antigen specific $\mathrm{T}$ cell immunity induced by the oral targeted mini gene-vaccine. One week after $\mathrm{x} 31$ infection, mice were sacrificed; lungs were perfused and homogenized in order to determine viral titers in this tissue by plaque assay. Our data indicate that FluVac-DC vaccinated mice had lower viral titers in the lungs, which suggests that induced antigen specific $\mathrm{T}$ cell was efficacious in a murine vaccine model (data not shown).

In conclusion, novel vaccine strategies that target conserved cytotoxic $\mathrm{T}$ lymphocyte (CTL) epitope regions of viral proteins are urgently needed. Accordingly, data show that various CTL epitopes are potentially present in viral genes that induce robust antiviral $\mathrm{CD} 8^{+} \mathrm{T}$ cell immune responses. The current scientific focus of our laboratory is the design of novel multivalent $\mathrm{CD} 4^{+}$and $\mathrm{CD} 8^{+} \mathrm{T}$ cell targeted vaccines for numerous influenza A strains that can be delivered orally in order to resist the viral challenge. Such a vaccination regimen is, indeed, effective at conferring immune protection against numerous viral infections, including influenza A in vivo. Therefore, our platform for introducing an oral targeted mini gene-vaccine platform that can establish optimal activation of Flu-antigen specific lung $\mathrm{T}$ cell immune responses against influenza A will provide a unique treatment strategy within the fields of both vaccination and therapy.

\section{Acknowledgments}

This work was supported in part by NIH Grant 1R01Al098833-01, and Emerging Pathogens Institute Funds. I wish to thank Dr. David Woodland for fruitful discussion, and Mrs. Mojgan Zadeh and Mrs. Rana Saber for excellent technical assistance.

\section{References}

1. Clark I, Alleva L (2009) Invited commentary on David Fedson's article. Influenza Other Respi Viruses 3: 199-201.

2. Sullivan SP, Koutsonanos DG, Del Pilar Martin M, Lee JW, Zarnitsyn V et al. (2010) Dissolving polymer microneedle patches for influenza vaccination. Nat Med 16: 915-920.

3. Erskine CL, Krco CJ, Hedin KE, Borson ND, Kalli KR, et al. (2011) MHC class II epitope nesting modulates dendritic cell function and improves generation of antigen-specific CD4 helper T cells. J Immunol 187: 316-324.

4. Mohamadzadeh M, Duong T, Sandwick SJ, Hoover T, Klaenhammer TR (2009) Dendritic cell targeting of Bacillus anthracis protective antigen expressed by Lactobacillus acidophilus protects mice from lethal challenge. Proc Natl Acad Sci USA 106: 4331-4336.

5. Mohamadzadeh M, Durmaz E, Zadeh M, Pakanati KC, Gramarossa M, et 
Citation: Mohamadzadeh M (2012) Novel Oral Targeted Mini Gene-Vaccine Platform Resists Influenza A Challenge. J Vaccines Vaccin 3:e112. doi:10.4172/2157-7560.1000e112

Page 3 of 3

al. (2010) Targeted expression of anthrax protective antigen by Lactobacillus gasseri as an anthrax vaccine. Future Microbiol 5: 1289-1296.

6. Mohamadzadeh M, Olson S, Kalina WV, Ruthel G, Demmin GL, et al. (2005) Lactobacilli activate human dendritic cells that skew $\mathrm{T}$ cells toward $\mathrm{T}$ helper 1 polarization. Proc Natl Acad Sci USA 102: 2880-2885.

7. Crowe SR, Miller SC, Woodland DL (2006) Identification of protective and nonprotective T cell epitopes in influenza. Vaccine 24: 452-456
8. Crowe SR, Turner SJ, Miller SC, Roberts AD, Rappolo RA et al. (2003) Differential antigen presentation regulates the changing patterns of CD8+ T cell immunodominance in primary and secondary influenza virus infections. J Exp Med 198: 399-410.

9. Cauley LS, Cookenham T, Hogan RJ, Crowe SR, Woodland DL (2003) Renewal of peripheral CD8+ memory T cells during secondary viral infection of antibodysufficient mice. J Immunol 170: 5597-5606. 